A Brief Note is a short paper that presents a specific solution of technical interest in mechanics but which does not necessarily contain new general methods or results. A Brief Note should not exceed 1500 words or equivalent (a typical one-column figure or table is equivalent to 250 words; a one line equation to 30 words). Brief Notes will be subject to the usual review procedures prior to publication. After approval such Notes will be published as soon as possible. The Notes should be submitted to the Technical Editor of the Journal of Applied Mechanics. Discussions on the Brief Notes should be addressed to the Editorial Department, ASME, United Engineering Center, 345 East 47th Street, New York, N. Y. 10017, or to the Technical Editor of the JouRnAL OF APPLIED Mechanics. Discussions on Brief Notes appearing in this issue will be accepted until two months after publication. Readers who need more time to prepare a Discussion should request an extension of the deadline from the Editorial Department.

\section{Cruciform Rigid Line Problem in an Infinite Plate}

\section{Y. Z. Chen ${ }^{1}$}

\section{Introduction}

Recently there has been a resurgence of interest in the rigid line problem. Many works in this field were presented by Wang, Zhang, and Chou (1985), Hasebe and Takeuchi (1985), Dunders and Markenscoff (1989), Dunders (1989), Ballarini (1990), Chen and Hasebe (1992a), and Markenscoff and Li (1996). Characteristics of the stress field near the tip of a rigid line inhomogeneity can be found from an earlier investigation by England (1971). England analyzed the singular behavior of a wedge with the wedge angle $\alpha=\pi$ when the two edges are fixed. The analysis invariably leads to the conclusion that the stresses are singular with the asymptotic expression $\sigma_{i j} \approx$ $O\left(r^{-1 / 2}\right)$, where $r$ denotes the distance between the rigid line tip and a point in the vicinity of the tip. The stress singularity coefficient (abbreviated as SSC) was defined and the leading term of stresses was also introduced by Hasebe and Takeuchi (1985).

For the simple reason that the definition used for SSC has the same expression with the crack problem, we use the following definition:

$$
K_{\mid R}-i K_{2 R}=2 \sqrt{2 \pi} \operatorname{Lim}_{z \rightarrow a} \sqrt{z-a} \phi^{\prime}(z)
$$

where the rigid line tip is located at the point $z=a$ and $\phi^{\prime}(z)$ is a complex potential defined by Muskhelishvili (1953).

In this note the cruciform rigid line problem is studied. The objective of this paper is to provide the singular integral equation approach for the problem. Secondly, numerical results are

\footnotetext{
${ }^{1}$ Laboratory for Nonlinear Mechanics of Continuous Media, Institute of Mechanics, Chinese Academy of Sciences, Beijing 100080, P. R. China. (Permanent address: Division of Engineering Mechanics, Jiangsu University of Science and Technology, Zhenjiang, Jiangsu 212013, P. R. China.)

Contributed by the Applied Mechanics Division of THE AMERICAN SOCIETY OF MECHANICAL ENGINEERS for publication in the ASME JouRnAL OF APPLIED MechanICs. Manuscript received by the ASME Applied Mechanics Division, Dec. 5, 1996; final revision, Aug. 14, 1997. Associate Technical Editor: J. N. Reddy.
}

present to show the interaction between rigid branches. In addition, some particular features in the rigid line problem are compared with those in the crack problem.

\section{Analysis}

The following analysis depends on the complex variable function method in plane elasticity (Muskelishvili, 1953). In this method the stresses $\left(\sigma_{x}, \sigma_{y}, \sigma_{x y}\right)$, the displacements $(u, v)$, and the resultant force function $(X, Y)$ are expressed in terms of two complex potentials $\phi(z), \psi(z)$ such that

$$
\begin{gathered}
\sigma_{x}+\sigma_{y}=4 \operatorname{Re} \phi^{\prime}(z) \\
\sigma_{y}-\sigma_{x}+2 i \sigma_{x y}=2\left[\bar{z} \phi^{\prime \prime}(z)+\psi^{\prime}(z)\right] \\
f=-Y+i X=\phi(z)+z \overline{\phi^{\prime}(z)}+\overline{\psi(z)} \\
2 G(u+i v)=\kappa \phi(z)-z \overline{\phi^{\prime}(z)}-\overline{\psi(z)}
\end{gathered}
$$

where $G$ is the shear modulus of elasticity, $\kappa=(3-\nu) /(1+$ $\nu$ ) for the plane stress problem, and $\nu$ is the Poisson's ratio. In addition, the following two derivatives in a specified direction are useful (Chen and Hasebe 1992b):

$$
\begin{aligned}
J_{1}(z, \overline{d z}) & =\operatorname{Lim}_{\Delta z \rightarrow 0} \frac{1}{\Delta z}\left\{(f)_{z+\Delta z}-(f)_{z}\right\} \\
& =\phi^{\prime}(z)+\overline{\phi^{\prime}(z)}+\frac{\overline{d z}}{d z}\left(z \overline{\phi^{\prime \prime}(z)}+\overline{\psi^{\prime}(z)}\right) \\
& =N+i T \\
J_{2}\left(z, \frac{\overline{d z}}{d z}\right)= & 2 G \operatorname{Lim}_{\Delta z \rightarrow 0} \frac{1}{\Delta z}\left\{(u+i v)_{z+\Delta z}-(u+i v)_{z}\right\} \\
= & \kappa \phi^{\prime}(z)-\overline{\phi^{\prime}(z)}-\frac{\overline{d z}}{d z}\left(z \overline{\phi^{\prime \prime}(z)}+\overline{\psi^{\prime}(z)}\right)
\end{aligned}
$$

Physically, the $J_{1}$ value represents the traction $N+i T$ applied along the interval $z, z+\Delta z$ in Fig. 1 .

In the analysis, the solution of the cruciform rigid line problem depends on the potential for a single rigid line problem. The appropriate complex potential for the single rigid line problem has been obtained previously by Chen and Hasebe (1992a), which is as follows: 


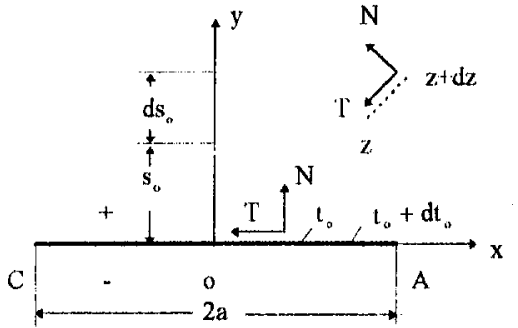

Fig. 1 A single rigid line problem in an infinite plate

$$
\begin{gathered}
\phi(z)=-\frac{1}{2 \pi} \int_{-a}^{a} \log (z-t) h(t) d t \\
\psi(z)=\frac{\kappa}{2 \pi} \int_{-a}^{a} \log (z-t) h(t) d t-\frac{1}{2 \pi} \int_{-a}^{a} \frac{t h(t) d t}{t-z}
\end{gathered}
$$

where $h(t), t \in(-a, a)$ takes the complex value in general. Physically, the function $h(t)$ represents the body force density. In this note only the normal mode case with the remote stresses $\sigma_{x}^{\infty}, \sigma_{y}^{\infty}$ is considered. In this case, the function $h(t)$ is an odd one and takes the real value.

Substituting (7) into (5) and (6), letting $z \rightarrow t_{0}^{+}$and $z \rightarrow$ $t_{0}^{-} t_{0} \in(-a, a)$ (Fig. 1), and using some results obtained previously (Chen and Hasebe, 1992b) along the line CA we can get

$$
\begin{aligned}
& {\left[J_{1}\left(t_{0}, 1\right)\right]^{+}-\left[J_{1}\left(t_{0}, 1\right)\right]^{-}=\left[N\left(t_{0}\right)+i T\left(t_{0}\right)\right]^{+}} \\
& -\left[N\left(t_{0}\right)+i T\left(t_{0}\right)\right]^{-}=i(\kappa+1) h\left(t_{0}\right), \quad\left|t_{0}\right|<a \\
& {\left[J_{2}\left(t_{0}, 1\right)\right]^{+}=\left[J_{2}\left(t_{0}, 1\right)\right]^{-}=J_{2}\left(t_{0}, 1\right)=\frac{\kappa}{\pi} \int_{-a}^{a} \frac{h(t) d t}{t-t_{0}},} \\
& \left|t_{0}\right|<a .
\end{aligned}
$$

Equation (8) shows that the $J_{1}$ contribution has a jump when a moving point is passing through the line $\mathrm{CA}$ in Fig. 1. On the contrary, Eq. (9) shows that the $J_{2}$ contribution is continuous in the same condition.

The $J_{2}$ contribution along the interval $\overline{i s_{0}, i\left(s_{0}+d s_{0}\right)}$ in Fig. 1 can be obtained by simply substituting $z=i s_{0}, d z=i d s_{0}$, $\frac{1}{d z} / d z=-1$, in (6), and it can be expressed in the form

$J_{2}=\frac{\kappa}{2 \pi} \int_{-a}^{a} \frac{2 i s_{0} h(t) d t}{t^{2}+s_{0}^{2}}-\frac{1}{2 \pi} \int_{-a}^{a} \frac{2 t h(t) d t}{\left(t+i s_{0}\right)^{2}}$

(for a point along the $y$-axis in Fig. 1).

Since the rigid line is in equilibrium, from Eq. (8) we obtain the following constraint equation:

$$
\int_{-a}^{a} h(t) d t=0
$$

From Eqs. (2) and (7), the SSC at the right tip A in Fig. 1 can be evaluated from

$$
K_{1 R}=-(2 \pi)^{1 / 2} \operatorname{Lim}_{t-a} \sqrt{|t-a|} h(t) .
$$

The original stress field for the cruciform rigid line problem (Fig. 2(a)) can be considered as a superposition of the uniform stress field (Fig. 2(b)) and the perturbation field (Fig. 2(c)). Clearly, the original stress field has the following solution (Muskhelishvili, 1953):

$$
2 G(u+i v)=(\kappa-1) \Gamma z-\Gamma_{1} \bar{z}
$$

where

$$
\Gamma=\left(\sigma_{x}^{\infty}+\sigma_{y}^{\infty}\right) / 4, \quad \Gamma_{1}=\left(\sigma_{y}^{\infty}-\sigma_{x}^{\infty}\right) / 2 .
$$

Actually, the $J_{2}$ contribution caused by the uniform stress field should be canceled by the $J_{2}$ contribution caused by the perturbation field. Secondly, the cruciform rigid line problem is considered as a superposition of two single rigid line problems. With this in mind, letting the body force density $h_{1}\left(s_{1}\right)$, $\left(h_{2}\left(s_{2}\right)\right)$ be placed along the horizontal portion (the vertical portion) of the cruciform configuration, and considering the $J_{2}$ contributions as shown by (9) and (10), we obtain the following system of singular integral equations:

$\frac{2 \kappa}{\pi} \int_{0}^{a} \frac{t h_{1}(t) d t}{t^{2}-s_{1}^{2}}+\frac{2}{\pi} \int_{0}^{b} \frac{t\left(s_{1}^{2}-t^{2}\right) h_{2}(t) d t}{\left(t^{2}+s_{1}^{2}\right)^{2}}=(1-\kappa) \Gamma+\Gamma_{1}$,

$$
\left(0<s_{1}<a\right)
$$

$\frac{2 \kappa}{\pi} \int_{0}^{b} \frac{t h_{2}(t) d t}{t^{2}-s_{2}^{2}}+\frac{2}{\pi} \int_{0}^{a} \frac{t\left(s_{2}^{2}-t^{2}\right) h_{1}(t) d t}{\left(t^{2}+s_{2}^{2}\right)^{2}}=(1-\kappa) \Gamma-\Gamma_{1}$,

$$
\left(0<s_{2}<b\right) \text {. }
$$

In the studied case, since the functions $h_{1}\left(s_{1}\right),\left(h_{2}\left(s_{2}\right)\right)$ are odd real functions, the condition like Eq. (11) is satisfied automatically. It is of interest to point out that substituting $\kappa=-1$ into Eqs. (15) and (16) will yield the singular integral equation for the cruciform crack problem (Boiko and Karpenko, 1981).

After solving the integral equation, the SSCs at two tips A and B (Fig. 2) can be evaluated by

$K_{1 R, A}=-(2 \pi)^{1 / 2} \operatorname{Lim}_{r \rightarrow a} \sqrt{|t-a|} h_{1}(t)$

(for the tip A in Fig. 2)

$K_{1 R, B}=-(2 \pi)^{1 / 2} \operatorname{Lim}_{t \rightarrow b} \sqrt{|t-b|} h_{2}(t)$

(for the tip B in Fig. 2).

\section{Numerical Example}

In the numerical solution, a semi-open quadrature rue is most suitable to solve the singular integral Eq. (15) and (16). The quadrature rule suggested by Boiko and Karpenko (1981) is used to solve the singular integral equation.

In the numerical solution we let

$$
\begin{array}{ll}
h_{1}(t) & =\sqrt{\frac{t}{a-t}} H_{1}(t), \quad(0 \leq t \leq a) \\
h_{2}(t) & =\sqrt{\frac{t}{b-t}} H_{2}(t), \quad(0 \leq t \leq b) .
\end{array}
$$

Finally, by using (17), (18), and (19) the SSCs at two rigid line tips $\mathrm{A}$ and $\mathrm{B}$ can be evaluated by

$$
K_{1 R, A}=-(2 \pi a)^{1 / 2} H_{1}(a), \quad K_{1 R, B}=-(2 \pi b)^{1 / 2} H_{2}(b) .
$$

Under the following conditions (1) the plane stress condition

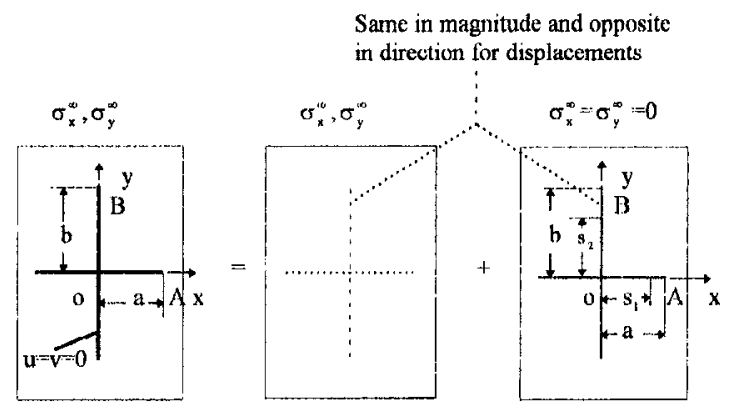

(a)

(b)

(c)

Fig. 2 Utilization of the superposition principle (a) the original stress field, (b) the uniform stress field, $(c)$ the perturbation stress field 


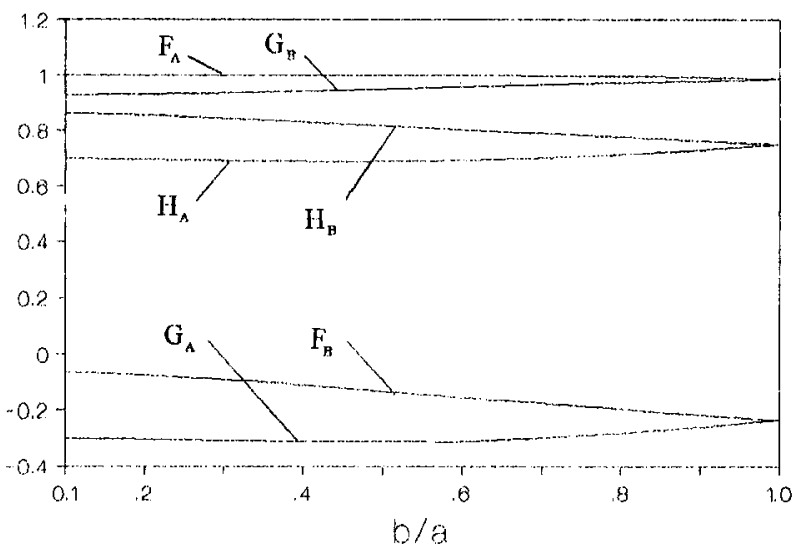

Fig. 3 Normalized SSCs at two rigid line tips A and B (see Fig. 2 and Eqs. (21), (22), (23), (24))

and $\nu=0.3$ are assumed, and (b) the remote stresses $\sigma_{x}^{\infty}, \sigma_{y}^{\infty}$ are applied at infinity. The SSCs at two rigid line tips are expressed by

$K_{1 R, A}=\left(F_{A}(b / a) \sigma_{x}^{\infty}+G_{A}(b / a) \sigma_{y}^{\infty}\right) \frac{\kappa+1}{4 \kappa} \sqrt{\pi a}$

(for the tip A in Fig. 2)

$K_{1 R, B}=\left(F_{B}(b / a) \sigma_{x}^{\infty}+G_{B}(b / a) \sigma_{y}^{\infty}\right) \frac{\kappa+1}{4 \kappa} \sqrt{\pi b}$

(for the tip B in Fig. 2).

Finally, the calculated $F_{A}(b / a), G_{A}(b / a), F_{B}(b / a), G_{B}(b / a)$ values are plotted in Fig. 3 .

In the case of $\sigma_{x}^{\infty}=\sigma_{y}^{\infty}=p$, the SSCs can be expressed by

$K_{1 R, A}=H_{A}(b / a) p \frac{\kappa+1}{4 \kappa} \sqrt{\pi a}, \quad H_{A}=F_{A}+G_{A}$

(for the tip A in Fig. 2)

$K_{1 R, B}=H_{B}(b / a) p \frac{\kappa+1}{4 \kappa} \sqrt{\pi b}, \quad H_{B}=F_{B}+G_{B}$

(for the tip B in Fig. 2).

The calculated results for $F_{A}, F_{B}, G_{A}, G_{B}, H_{A}, H_{B}$ values are listed plotted in Fig. 3. From Fig. 3 we see that, in the remote stress $\sigma_{x}^{\infty}$ case, $F_{A}$ values are dominant and $\left|F_{A}\right|>\left|F_{B}\right|$. In the meantime, in the remote stress $\sigma_{y}^{\infty}$ case, $G_{B}$ values are dominant and $\left|G_{B}\right|>\left|G_{A}\right|$.

\section{Remarks}

A comparison has been made between the rigid inclusion problem with the cavity problem (Dunders, 1989). Similarly, we also make a comparison for two problems. In the cruciform crack case with the remote stress $\sigma_{x}^{\infty}$, the stress intensity factors at the tips A and B can be expressed by (Chen, 1993)

Table 1 Comparison results for the cruciform rigid line problem and the cruciform crack problem

\begin{tabular}{lrrr}
\hline & \multicolumn{3}{c}{$b / a=$} \\
\cline { 2 - 4 } & \multicolumn{1}{c}{0.1} & 0.2 & \multicolumn{1}{c}{1.0} \\
\hline$F_{A}$ & 1.000 & 1.000 & 0.985 \\
$F_{B}$ & -0.064 & -0.075 & -0.235 \\
$f_{A}$ & 0.003 & 0.001 & -0.217 \\
$f_{B}$ & 0.997 & 0.998 & 1.081 \\
\hline
\end{tabular}

$$
K_{1 A}=f_{A}(b / a) \sigma_{x}^{\infty} \sqrt{\pi a}, \quad K_{1 B}=f_{B}(b / a) \sigma_{x}^{\infty} \sqrt{\pi b} .
$$

The calculated $F_{A}, F_{B}$ (for the cruciform rigid line problem) and $f_{A}, f_{B}$ (for the cruciform crack problem) values are listed in Table 1. From Table 1 we see that in the cruciform rigid line case $F_{A}$ values are dominant and $\left|F_{A}\right|>\left|F_{B}\right|$, and in the cruciform crack case $f_{B}$ values are dominant and $\left|f_{B}\right|>\left|f_{A}\right|$.

\section{Acknowledgment}

The research project is support by National Natural Science Fund of China.

\section{References}

Ballarini, R., 1990, "A Rigid Line Inclusion at a Bimeterial Interface," Engineering Fracture Mechanics, Vol. 37, pp. 1-5.

Boiko, A. V., and Karpenko, L. N., 1981, "On Some Numerical Methods for the Solution of Plane Elasticity Problems for Bodies with Cracks by Means of Singular Integral Equations," International Journal of Fracture, Vol. 17, pp. 381-388.

Chen, Y. Z., and Hasebe, N., 1992a, "Integral Equation Approaches for Curved Rigid Line Problem in an Infinite Plate," International Journal of Fracture, $\mathrm{Vol}_{\text {. }}$ 58 , pp. $1-20$.

Chen, Y.Z., and Hasebe, N., 1992b, "Interaction of Two Curved Cracks in an Infinite Plate," Archive of Applied Mechanics, Vol. 62, pp. 147-157.

Chen, Y.Z., 1993, "Numerical Solution for a Cruciform Crack Problem," International Journal of Fracture, Vol. 63, pp. R31-R34.

Dundurs, J., and Markenscoff, X., 1989, “A Green's Function Formulation of Anticracks and Their Interaction with Load-induced Singularities," ASME JourNaL of Applied Mechanics, Vol. 56, pp. 550-555.

Dundurs, J., 1989, "Cavities vis-a-vis Rigid Inclusion and Some Related General Results in Plane Elasticity," ASME JouRnal of APPLIED MECHANICS, Vol. 56, pp. $786-790$

England, A. H., 1971, "On Stress Singularities in Linear Elasticity," International Journal of Engineering Science, Vol. 9, pp. 571-585.

Hasebe, N., and Takeuchi, T., 1985, "Stress Analysis a Semi-infinite Plate with a Thin Rigid Body," International Journal of Engineering Science, Vol. 23, pp. 531-539.

Markenscoff, X., and Li, N., 1996, "The Debonded Interface Anticrack," ASME JoURNAL OF APPLIED MECHANICs, Vol. 63, pp. 62l-627.

Muskhelishvili, N. I, 1953, Some Basic Problems in the Theory of Elasticity, Noorhoff, Leyden, The Netherlands.

Wang, Z. Y., Zhang, H. T., and Chou, Y. T., 1985, "Characteristics of Elastic Field of a Rigid line Inhomogeneity," ASME Journal of APPLIED MEChanICs, Vol. 52, pp. $818-822$.

\section{A Modified Element-by-Element Preconditioner for Elastostatics}

\section{Barboteu ${ }^{2}$, P. Alart ${ }^{2}$, and F. Lebon ${ }^{2}$}

In this paper we present in detail a modified element-by-element strategy. This kind of method is well known to be effective for large-scale problems because of its implicit parallelism. Numerical experiments described in this paper confirm the efficiency of this solver.

\section{Introduction}

Hughes, Levit, and Winget (1983) and also Hughes and Winget (1985) have introduced an element-by-element precon-

\footnotetext{
${ }^{2}$ Laboratoire de Mécanique et Génie Civil, UMR 5508 Université Montpellier 2.CNRS, Pl. E. Bataillon, 34095 Montpellier Cedex 5, France.

Contributed by the Applied Mechanics Division of THE AMERICAN SOCIETY of Mechanical Engineers for publication in the ASME Journal of APPLiEd MeChanics, Manuscript received by the ASME Applied Mechanics Division, Feb. 27, 1997; final revision, Aug. 11, 1997. Associate Technical Editor: W. K, Liu.
} 